\title{
Tratamento da neuralgia pós-herpética com irradiações a laser de baixa intensidade: revisão da literatura
}

\author{
Treatment of postherpetic neuralgia with low-level laser irradiation: \\ literature review
}

\author{
Raimundo Nonato Silva Gomes ${ }^{1} \bowtie$, Vânia Thais Silva Gomes ${ }^{1}$, Renata Amadei Nicolau, 2 \\ Programa de Pós-Graduação em Engenharia Biomédica do Instituto de Pesquisa e Desenvolvimento da Universidade do Vale do Paraíba (UNIVAP). \\ São José dos Campos, SP. \\ 2 Programa de Mestrado Profissional em Bioengenharia do Instituto de Pesquisa e Desenvolvimento da UNIVAP. São José dos Campos, SP.
}

\section{RESUMO}

Objetivos: Realizar uma revisão da literatura sobre o tratamento da neuralgia pós-herpética com irradiações a laser de baixa intensidade. Métodos: A revisão foi realizada nos meses de novembro a dezembro de 2015 nas bases de dados LILACS, SciELO e Medline, utilizando-se os descritores "herpes zoster", "neuralgia" e "terapia a laser de baixa intensidade" e seus correspondentes termos em inglês. Os critérios de inclusão foram artigos disponíveis na íntegra, publicados em português, inglês, alemão ou espanhol, no período compreendido entre 2008 a 2015. Os critérios de exclusão foram textos incompletos, artigos que não abordaram diretamente a temática do estudo e repetição de um mesmo artigo na mesma ou em diferentes bases de dados, nesse caso sendo considerado apenas um deles.

Resultados: A partir dos descritores utilizados foram encontrados 32 estudos. Após aplicação dos critérios de elegibilidade foram selecionados 15 artigos para leitura, dos quais foram excluídos dois por repetição e um por não apresentar resultados relevantes a esta pesquisa. Dessa forma, 11 estudos foram incluídos na revisão, dos quais cinco foram realizados nos Estados Unidos, dois no Brasil, dois na Alemanha e dois na Itália, sendo quatro ensaios clínicos, duas revisões sistemáticas e cinco relatos de casos. Os 11 estudos encontraram resultados positivos após aplicação do laser, principalmente diminuição da dor, e apenas um artigo mencionou pouca redução da neuralgia. Nos três estudos que informaram os parâmetros de irradiação estes não foram uniformes, sendo a média do comprimento de onda 740,6 nm, a média da potência $28 \mathrm{~mW}$ e a média do tempo de irradiação por ponto 53,4 segundos. Quanto ao número de sessões, houve grande variação, com uma média de aproximadamente 12 sessões em dia alternados.

Conclusões: A terapia com laser de baixa intensidade ofereceu resultados positivos aos pacientes com neuralgia pós-herpética, entretanto não há um consenso quanto aos parâmetros a serem utilizados. Em razão dos diferentes protocolos descritos, é importante realizar mais estudos que auxiliem a padronizar os parâmetros dessa terapia aplicados à neuralgia pós-herpética.

DESCRITORES: herpes zoster; neuralgia; terapia a laser de baixa intensidade.

\section{ABSTRACT}

Aims: To perform a literature review on the treatment of postherpetic neuralgia with low-intensity laser irradiation.

Methods: The review was conducted in November and December 2015 by searching the terms "herpes zoster", "neuralgia", "low-level laser therapy", and "low-intensity laser therapy" in the LILACS, SciELO, and Medline databases. Full articles published in Portuguese, English, German, or Spanish between 2008 and 2015 were included. Incomplete articles, those that did not directly address the topic of interest, and those appearing in the same or in different databases were excluded. In the latter case, only one article was included.

Results: Thirty-two studies were selected using the search terms. After the application of the eligibility criteria, 15 studies were read; two of them were excluded for being available in more than one database and one was eliminated for not adding relevant information. Therefore, 11 studies were included in the review: five of them conducted in the United States, two in Brazil, two in Germany, and two in Italy. There were four clinical trials, two systematic reviews and five case reports. The 11 studies found positive results after laser application, especially reduction of pain, with only one article mentioning little reduction of neuralgia. In the three studies that reported the irradiation parameters, these have not been uniform, with a medium wavelength of $740.6 \mathrm{~nm}$, a medium power of $28 \mathrm{~mW}$ and a medium time of irradiation per point of 53.4 seconds. There was great variation in the number of sessions, with an average of about 12 sessions on alternate days.

Conclusions: The low-intensity laser therapy provided positive results to patients with postherpetic neuralgia, however there is no consensus about the parameters to be used. In view of the different protocols, it is important to perform new studies in order to standardize the lowintensity laser therapy parameters applied to postherpetic neuralgia.

KEY WORDS: herpes zoster; neuralgia; laser therapy, low-level; low-intensity laser therapy.

Recebido: fevereiro, 2016

Aceito: abril, 2016

Este artigo está licenciado sob forma de uma licença Creative Common Atribuição 4.0 Internacional, que permite uso irrestrito, distribuição e reprodução em qualuer meio, desde que a publicação original seja corretamente citada. 
Abreviaturas: LBI, laser de baixa intensidade; HZ, herpes zoster; $\mathrm{NPH}$, neuralgia pós-herpética.

\section{INTRODUÇÃO}

O herpes zoster (HZ) é ocasionado pelo vírus Varicella-zoster, um herpesvírus da subfamília Alphaherpesvirinae, gênero Varicellovirus, cuja característica mais marcante é a capacidade de estabelecer latência. Suas partículas medem cerca de 180 a $200 \mathrm{~nm}$ de diâmetro e têm forma esférica ou poligonal. A reativação do vírus Varicella-zoster está intimamente relacionada ao sistema imunológico, uma vez que o $\mathrm{HZ}$ ocorre principalmente em indivíduos imunocomprometidos por doenças como câncer e aids, assim como também em situações como imunossupressão pós-transplante, quimioterapia e idade avançada, na qual há um declínio na resposta imune mediada pelas células T. A idade avançada é o fator de risco mais importante para $\mathrm{HZ}$, sendo o aumento da incidência da doença mais marcante acima dos 55 anos [1,2].

Clinicamente o HZ apresenta-se com lesões vesiculares na pele, precedidas ou seguidas de algias neurais, além de mal-estar geral, prurido nos locais acometidos e sensações de dormência e formigamento. As erupções cutâneas ocorrem no trajeto cutâneo do nervo acometido (dermátomo) de um lado do corpo, formando uma faixa $[3,4]$. A neuralgia pós-herpética (NPH), sintoma mais comum em pacientes acometidos por HZ, é caracterizada por dor neuropática crônica no trajeto do nervo afetado, que se inicia entre um e seis meses após a cura das erupções cutâneas, podendo durar de meses a anos [1].

Diante disso, o uso terapêutico do laser de baixa intensidade (LBI) surge como uma nova abordagem terapêutica à NPH. O LBI tem sido bastante empregado na prática clínica de diversas áreas da saúde, devido aos seus efeitos anti-inflamatório, analgésico, antiedematoso e de contribuição para o reparo tecidual. Entre os efeitos citados pode-se incluir também a degranulação de mastócitos, o aumento da circulação periférica, a vasodilatação e a proliferação fibroblástica [5]. Muitas pesquisas, in vitro e in vivo, vêm sendo realizadas sobre LBI e seu uso terapêutico. Resultados favoráveis têm sido descritos, embora alguns estudos demonstrem o contrário em alguns usos específicos [6,7].

Diante disso, o presente trabalho teve como objetivo realizar uma revisão da literatura sobre o tratamento da NPH com irradiações a LBI.

\section{MÉTODOS}

Para a elaboração da presente revisão, foram seguidos os seguintes procedimentos metodológicos: formulação dos objetivos da revisão; estabelecimento de critérios de inclusão e exclusão; categorização dos estudos; avaliação dos estudos incluídos na pesquisa; e apresentação dos resultados. A seleção do material foi realizada nos meses de novembro a dezembro de 2015. Para a seleção dos artigos foram utilizadas três bases de dados eletrônicas: LILACS (Literatura Latino Americana e do Caribe em Ciências Sociais e da Saúde), SciELO (Scientific Electronic Library Online) e Medline (Medical Literature Analysis and Retrieval System Online). Os descritores foram selecionados por meio de consultas no DeCS (Descritores em Ciências da Saúde) e MeSH (Medical Subject Headings), sendo eles: "herpes zoster", "neuralgia" e "terapia a laser de baixa intensidade", assim como os termos em inglês "low-level laser therapy", and "low-intensity laser therapy".

Os critérios de inclusão foram artigos disponíveis na íntegra, publicados em português, inglês, alemão ou espanhol, no período compreendido entre 2008 a 2015. Os critérios de exclusão foram textos incompletos, artigos que não abordassem diretamente a temática do estudo ou que não abordassem resultados relevantes à pesquisa, e repetição de um mesmo artigo na mesma base ou em diferentes bases de dados, nesse caso considerando-se apenas um deles.

\section{RESULTADOS DA SELEÇÃO}

A partir dos descritores utilizados, obtiveram-se 32 publicações, sendo 21 no Medline, oito no LILACS e três no SciELO. Após aplicação dos critérios de elegibilidade foram selecionados 15 artigos para leitura, dos quais foram excluídos dois por repetição e dois por não apresentar resultados relevantes a esta pesquisa. Dessa forma, 11 estudos foram incluídos na revisão, dos quais cinco foram realizados nos Estados Unidos, dois no Brasil, dois na Alemanha e dois na Itália (Figura 1 e Tabela 1).

Dos 11 artigos, dois foram publicados em 2009, um em 2010, um em 2011, quatro em 2012, dois em 2013 e um em 2014. Quanto ao idioma de publicação dos artigos analisados, oito $(72,6 \%)$ estão em Inglês, dois (18,2\%) em Português e um $(9,1 \%)$ em Alemão. Em relação aos delineamentos, foram quatro ensaios clínicos, sendo três randomizados, duas revisões sistemáticas e cinco relatos de casos. 


\section{CONTEÚDO DA REVISÃO}

A Tabela 1 apresenta as características dos estudos e dos protocolos utilizados, assim como os resultados do tratamento. Todos os estudos analisados encontraram eficácia das irradiações com LBI no tratamento da $\mathrm{NPH}$, chegando à conclusão de que essa terapia pode ser utilizada como terapia complementar. Apenas um estudo randomizado [15] mencionou pouca redução da neuralgia, e nenhum dos artigos, incluindo os ensaios clínicos, relatou efeitos colaterais indesejáveis. Tendo em vista que em muitos casos a terapêutica convencional com medicação é falha e não oferece qualidade de vida ao paciente, e podendo a neuralgia durar de meses a anos, a terapia a LBI mostra-se relevante.

No que se refere à forma de aplicação, $100 \%$ utilizaram contato direto do laser com a pele, de forma periférica ao nervo acometido na maioria dos casos. Em relação à escolha dos parâmetros de irradiação, oito $(72,6 \%)$ entre os estudos analisados não os descreveram. Nos três $(27,4 \%)$ estudos que informaram os parâmetros de irradiação, a média do comprimento de onda utilizado foi de $740,6 \mathrm{~nm}$; da potência foi $28 \mathrm{~mW}$; e a média do tempo de irradiação por ponto foi de 53,4 segundos. Quanto ao número de sessões de irradiação, houve grande variação, com uma média de aproximadamente 12 sessões, em dia alternados.

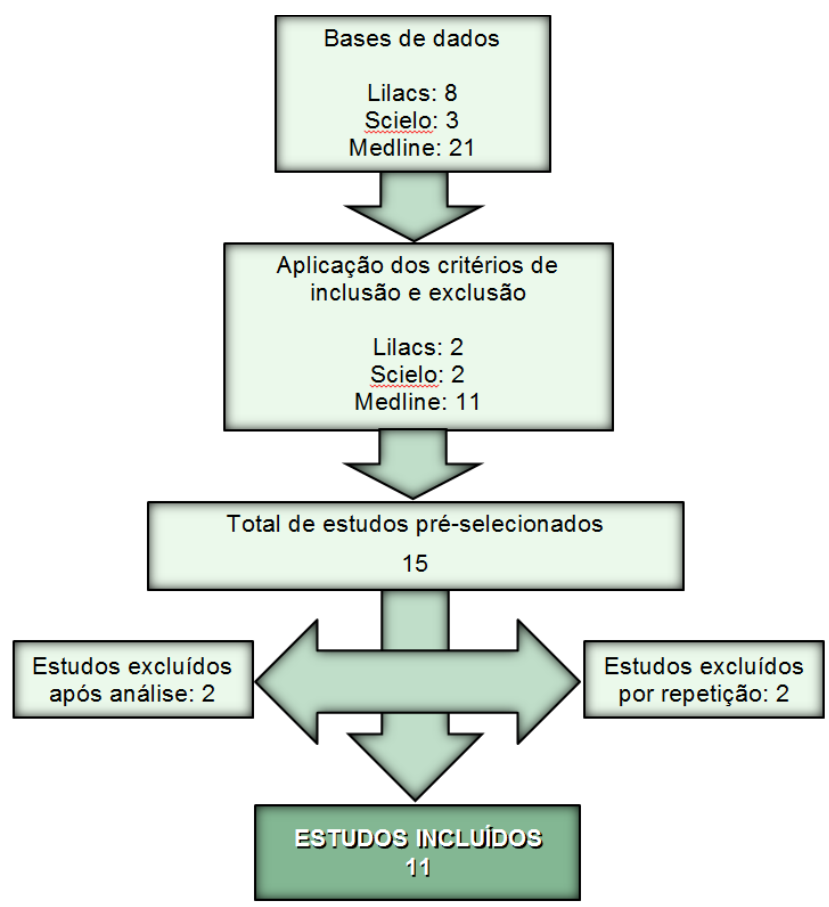

Figura 1. Fluxograma de seleção dos estudos utilizados na revisão de literatura sobre o tratamento da neuralgia pós-herpética com irradiações a laser de baixa intensidade.

Tabela 1. Artigos incluídos na revisão, organizados em ordem cronológica de publicação: autores, ano, país de origem, idioma, delineamento do estudo, parâmetros, número de sessões, tipos de laser, resultados e métodos de avaliação

\begin{tabular}{|c|c|c|c|c|c|c|c|}
\hline $\begin{array}{l}\text { Autores } \\
\text { Ano }\end{array}$ & $\begin{array}{c}\text { País de origem/ } \\
\text { idioma }\end{array}$ & $\begin{array}{l}\text { Delineamento } \\
\text { do estudo }\end{array}$ & $\begin{array}{l}\text { Forma de } \\
\text { irradiação }\end{array}$ & Parâmetros & Número de sessões & $\begin{array}{l}\text { Tipo de } \\
\text { laser }\end{array}$ & $\begin{array}{c}\text { Resultados e } \\
\text { métodos de avalição }\end{array}$ \\
\hline $\begin{array}{l}\text { Vasconcelos Filho PO [8] } \\
2012\end{array}$ & $\begin{array}{l}\text { Brasil/ } \\
\text { Português }\end{array}$ & $\begin{array}{l}\text { Relato de caso, } \\
\text { análise do antes } \\
\text { e depois das } \\
\text { irradiações com } \\
\text { laser. }\end{array}$ & $\begin{array}{l}\text { Contato direto } \\
\text { (ao redor do } \\
\text { nervo) }\end{array}$ & $\begin{array}{l}\lambda: 780 \mathrm{~nm} \\
\text { P: } 40 \mathrm{~mW} \\
\text { TI: } 40 \mathrm{~s} \\
\text { EP: } 4 \mathrm{~J} / \mathrm{cm}^{-2} \\
\text { DE: } 40 \mathrm{~J} / \mathrm{cm}^{2} \\
\text { DP: } \mathrm{NC} ; \\
\text { FQ: NC; } \\
\text { SAEF: } 15 \mathrm{~J} / \mathrm{cm}^{2}\end{array}$ & 14 (dias alternados) & $\begin{array}{l}\text { AlGalnP - } \\
\text { luz contínua }\end{array}$ & $\begin{array}{l}\downarrow \text { edema, TNF } \alpha \text {, } \\
\text { cinina, dor } \\
\uparrow \text { microcirculação }\end{array}$ \\
\hline $\begin{array}{l}\text { Abreu EMC et al. [9] } \\
2015\end{array}$ & $\begin{array}{l}\text { Brasil/ } \\
\text { Português }\end{array}$ & $\begin{array}{l}\text { Revisão } \\
\text { sistemática de } \\
\text { ensaios clínicos } \\
\text { controlados. }\end{array}$ & $\begin{array}{l}\text { Contato direto } \\
\text { (sobre o nervo) }\end{array}$ & $\begin{array}{l}\lambda: 904 \mathrm{~nm} \\
\text { P: } 20 \mathrm{~mW} \\
\text { TI: } 30 \mathrm{~s} \\
\text { EP: NC } \\
\text { DE: NC } \\
\text { DP: NC } \\
\text { FQ: NC } \\
\text { SAEF: NC }\end{array}$ & 40 (dias consecutivos) & NC & $\begin{array}{l}\downarrow \text { edema, PGE2, dor } \\
\uparrow \text { microcirculação }\end{array}$ \\
\hline $\begin{array}{l}\text { Chung H et al. [10] } \\
2012\end{array}$ & $\begin{array}{l}\text { Estados Unidos/ } \\
\text { Inglês }\end{array}$ & $\begin{array}{l}\text { Ensaio clínico } \\
\text { randomizado } \\
\text { controlado. }\end{array}$ & $\begin{array}{l}\text { Contato direto } \\
\text { (ao redor do } \\
\text { nervo) }\end{array}$ & $\begin{array}{l}\lambda: 810 \mathrm{~nm} \\
\text { P: } 41 \mathrm{~mW} \\
\text { TI: } 1,25 \mathrm{~s} \\
\text { EP: NC } \\
\text { DE: NC } \\
\text { DP: NC } \\
\text { FQ: NC } \\
\text { SAEF: NC }\end{array}$ & 8 (uma por semana) & $\mathrm{NC}$ & $\begin{array}{l}\uparrow \text { microcirculação } \\
\downarrow \text { dor }\end{array}$ \\
\hline
\end{tabular}


Tabela 1 (Conclusão)

\begin{tabular}{|c|c|c|c|c|c|c|c|}
\hline $\begin{array}{l}\text { Autores } \\
\text { Ano }\end{array}$ & $\begin{array}{l}\text { País de origem/ } \\
\text { idioma }\end{array}$ & $\begin{array}{l}\text { Delineamento } \\
\text { do estudo }\end{array}$ & $\begin{array}{l}\text { Forma de } \\
\text { irradiação }\end{array}$ & Parâmetros & Número de sessões & $\begin{array}{l}\text { Tipo de } \\
\text { laser }\end{array}$ & $\begin{array}{c}\text { Resultados e } \\
\text { métodos de avalição }\end{array}$ \\
\hline $\begin{array}{l}\text { Knapp DJ [11] } \\
2013\end{array}$ & $\begin{array}{l}\text { Estados Unidos/ } \\
\text { Inglês }\end{array}$ & $\begin{array}{l}\text { Relato de caso, } \\
\text { análise do antes } \\
\text { e depois das } \\
\text { irradiações com } \\
\text { laser em quatro } \\
\text { casos clínicos. }\end{array}$ & $\begin{array}{l}\text { Contato direto } \\
\text { (ao redor do } \\
\text { nervo) }\end{array}$ & $\begin{array}{l}\lambda: 810 \mathrm{~nm} \\
\text { P: NC } \\
\text { TI: NC } \\
\text { EP: } 7 \mathrm{~J} / \mathrm{cm}^{-2} \\
\text { DE: } 7,1 \mathrm{~J} / \mathrm{cm}^{2} \\
\text { DP: NC } \\
\text { FQ: NC } \\
\text { SAEF: } 20 \mathrm{~J} / \mathrm{cm}^{2}\end{array}$ & 8 (dias alternados) & $\begin{array}{l}\text { GaAlAs - } \\
\text { luz pulsada }\end{array}$ & $\begin{array}{l}\downarrow \text { dor } \\
\uparrow \text { qualidade de vida }\end{array}$ \\
\hline $\begin{array}{l}\text { Kneebone WJ [12] } \\
2012\end{array}$ & $\begin{array}{l}\text { Estados Unidos/ } \\
\text { Inglês }\end{array}$ & $\begin{array}{l}\text { Relato de caso, } \\
\text { análise do antes } \\
\text { e depois das } \\
\text { irradiações com } \\
\text { laser. }\end{array}$ & $\begin{array}{l}\text { Contato direto } \\
\text { (ao redor do } \\
\text { nervo) }\end{array}$ & $\begin{array}{l}\lambda: 904 \mathrm{~nm} \\
\text { P: } 40 \mathrm{~mW} \\
\text { TI: } 60 \mathrm{~s} \\
\text { EP: NC } \\
\text { DE: NC } \\
\text { DP: NC } \\
\text { FQ: NC } \\
\text { SAEF: NC }\end{array}$ & 10 (dias alternados) & $\begin{array}{l}\text { GaAs - } \\
\text { luz pulsada }\end{array}$ & $\begin{array}{l}\downarrow \text { células } \\
\text { inflamatórias e dor }\end{array}$ \\
\hline $\begin{array}{l}\text { Stump JL et al. [13] } \\
2011\end{array}$ & $\begin{array}{l}\text { Estados Unidos/ } \\
\text { Inglês }\end{array}$ & $\begin{array}{l}\text { Relato de caso, } \\
\text { análise do antes } \\
\text { e depois das } \\
\text { irradiações com } \\
\text { laser. }\end{array}$ & $\begin{array}{l}\text { Contato direto } \\
\text { (pontos de } \\
\text { acupuntura) }\end{array}$ & $\begin{array}{l}\lambda: \mathrm{NC} \\
\text { P: NC } \\
\text { TI: } 50 \mathrm{~s} \\
\text { EP: } 10 \mathrm{~J} / \mathrm{cm}^{-2} \\
\text { DE: NC } \\
\text { DP: NC } \\
\text { FQ: NC } \\
\text { SAEF: NC }\end{array}$ & 12 (dias alternados) & $\begin{array}{l}\text { GaAs - } \\
\text { luz pulsada }\end{array}$ & $\begin{array}{l}\uparrow \text { qualidade de vida } \\
\downarrow \text { dor }\end{array}$ \\
\hline $\begin{array}{l}\text { Valeriani } M \text { et al. [14] } \\
2012\end{array}$ & $\begin{array}{l}\text { Itália/ } \\
\text { Inglês }\end{array}$ & $\begin{array}{l}\text { Ensaio clínico } \\
\text { controlado } \\
\text { randomizado. }\end{array}$ & $\begin{array}{l}\text { Contato direto } \\
\text { (ao redor do } \\
\text { nervo) }\end{array}$ & $\begin{array}{l}\lambda: \text { NC } \\
\text { P: NC } \\
\text { TI: NC } \\
\text { EP: NC } \\
\text { DE: NC } \\
\text { DP: NC } \\
\text { FQ: NC } \\
\text { SAEF: NC }\end{array}$ & NC & $\begin{array}{l}\text { GaAlAs - } \\
\text { luz contínua }\end{array}$ & $\begin{array}{l}\downarrow \text { edema, histaminas, } \\
\text { PGE2, TNF } \alpha \\
\uparrow \text { adenosina }\end{array}$ \\
\hline $\begin{array}{l}\text { Franz M et al. [15] } \\
2012\end{array}$ & $\begin{array}{l}\text { Estados Unidos/ } \\
\text { Inglês }\end{array}$ & $\begin{array}{l}\text { Ensaio clínico } \\
\text { com } 16 \text { casos, } \\
\text { avaliando o antes } \\
\text { e depois das } \\
\text { irradiações com } \\
\text { laser. }\end{array}$ & $\begin{array}{l}\text { Contato direto } \\
\text { (sobre o nervo) }\end{array}$ & $\begin{array}{l}\lambda: \mathrm{NC} \\
\text { P: NC } \\
\text { TI: } 12,5 \mathrm{~s} \\
\text { EP: } 4,8 \mathrm{~J} / \mathrm{cm}^{-2} \\
\text { DE: NC } \\
\text { DP: NC } \\
\text { FQ: NC } \\
\text { SAEF: NC }\end{array}$ & 10 (dias consecutivos) & $\begin{array}{l}\mathrm{HeNe}- \\
\text { luz pulsada }\end{array}$ & $\begin{array}{l}\downarrow \text { cininas, células } \\
\text { inflamatórias e dor }\end{array}$ \\
\hline $\begin{array}{l}\text { Schafers } M \text { et al. [16] } \\
2013\end{array}$ & $\begin{array}{l}\text { Alemanha/ } \\
\text { Alemão }\end{array}$ & $\begin{array}{l}\text { Relato de caso } \\
\text { clínico, avaliando } \\
\text { a terapia } \\
\text { convencional e } \\
\text { a terapia com } \\
\text { laser de baixa } \\
\text { intensidade. }\end{array}$ & $\begin{array}{l}\text { Contato direto } \\
\text { (sobre o nervo) }\end{array}$ & $\begin{array}{l}\lambda: \text { NC } \\
\text { P: NC } \\
\text { TI: NC } \\
\text { EP: NC } \\
\text { DE: NC } \\
\text { DP: NC } \\
\text { FQ: NC } \\
\text { SAEF: NC }\end{array}$ & 10 (dias alternados) & $\mathrm{NC}$ & $\begin{array}{l}\uparrow \text { qualidade vida } \\
\downarrow \text { dor }\end{array}$ \\
\hline $\begin{array}{l}\text { Cruccua G et al. [17] } \\
2010\end{array}$ & $\begin{array}{l}\text { Itália/ } \\
\text { Inglês }\end{array}$ & $\begin{array}{l}\text { Revisão } \\
\text { sistemática de } \\
\text { ensaios clínicos } \\
\text { controlados } \\
\text { randomizados. }\end{array}$ & $\begin{array}{l}\text { Contato direto } \\
\text { (sobre o nervo) }\end{array}$ & $\begin{array}{l}\lambda: 750 \mathrm{~nm} \\
\text { P: } 21 \mathrm{~mW} \\
\text { TI: } 70 \mathrm{~s} \\
\text { EP: NC } \\
\text { DE: NC } \\
\text { DP: NC } \\
\text { FQ: NC } \\
\text { SAEF: } 18 \mathrm{~J} / \mathrm{cm}^{2}\end{array}$ & 15 (consecutivas) & $\begin{array}{l}\mathrm{CO}_{2}-\mathrm{luz} \\
\text { fracionada }\end{array}$ & $\begin{array}{l}\downarrow \text { edema e dor } \\
\uparrow \text { microcirculação }\end{array}$ \\
\hline $\begin{array}{l}\text { Fleckenstein J et al. [18] } \\
2009\end{array}$ & $\begin{array}{l}\text { Alemanha/ } \\
\text { Inglês }\end{array}$ & $\begin{array}{l}\text { Ensaio clínico } \\
\text { controlado } \\
\text { randomizado } \\
\text { realizado com } \\
336 \text { pacientes. }\end{array}$ & $\begin{array}{l}\text { Contato direto } \\
\text { (ponto de } \\
\text { acupuntura) }\end{array}$ & $\begin{array}{l}\lambda: 632 \mathrm{~nm} \\
\text { P: } 3 \mathrm{~mW} \\
\text { TI: } 35 \mathrm{~s} \\
\text { EP: NC } \\
\text { DE: NC } \\
\text { DP: NC } \\
\text { FQ: NC } \\
\text { SAEF: NC }\end{array}$ & 12 (dias alternados) & NC & $\begin{array}{l}\uparrow \text { microcirculação, } \\
\text { qualidade de vida } \\
\downarrow \text { edema e dor }\end{array}$ \\
\hline
\end{tabular}

NC, não consta; $\lambda$, comprimento de onda; P, Potência; TI, tempo de irradiação; EP, energia por ponto; DE, densidade de energia; DP, densidade de potência; FQ, frequência; SAEF, fluência de energia média espacial; $\downarrow=$ redução; $\uparrow=$ aumento;

AlGalnP, alumínio-Gálio-Índio-Fósforo; GaAlAs, arsenieto de gálio-alumínio; GaAs, arsenieto de gálio; HeNe, hélio neônio; $\mathrm{CO}_{2}$, dióxido de carbono. 


\section{DISCUSSÃO}

A terapia com LBI vem sendo largamente utilizada em vários países para o tratamento de dores agudas e crônicas. Atualmente, o funcionamento do laser está muito bem descrito, sendo o seu mecanismo de ação relacionado à melhora da circulação local e à inibição de agentes inflamatórios, além da estimular a produção de energia pela célula e liberação de neurotransmissores. Outra vantagem do LBI é ser um tratamento não invasivo, que garante ao paciente maior tranquilidade quanto à utilização da técnica $[8,9]$.

A terapia com LBI é classificada como um tratamento completamente seguro, uma vez que não há relatos conhecidos de efeitos deletérios relacionados a esse tipo de irradiação. O laser não possui a capacidade de aquecer, portanto a indução de lesões teciduais não é conhecida. O aprofundamento dos estudos na terapia com LBI possibilitou o desenvolvimento de diversos tipos de laser com variadas aplicações [7,10-12].

A terapia com LBI pode induzir alterações da permeabilidade da membrana ao cálcio e alterar o estado de oxidação celular. Essas alterações podem levar a mudanças na atividade celular e consequentemente em seus processos, como transcrição, tradução, proliferação celular e fagocitose. Vale salientar que essas alterações são dose-dependentes, considerando-se que doses entre $0,01 \mathrm{e} 10 \mathrm{~J} / \mathrm{cm}^{2}$ estimulam a atividade celular, enquanto doses maiores que $10 \mathrm{~J} / \mathrm{cm}^{2}$ a inibem $[9,13]$.

A terapia com LBI pode promover uma resposta anti-inflamatória por meio de um mecanismo que envolve a inibição de ácido araquidônico, reduzindo assim a expressão da cicloxigenase-2 (COX-2) e diminuindo a produção de prostaglandina E2, além de fatores de modulação ligados ao ácido ribonucleico de citocinas proinflamatórias. Por isso, a terapia com LBI pode melhorar o processo inflamatório provocado pelo HZ $[10,14,15]$. O comprimento de onda comumente utilizado varia de 600 a $900 \mathrm{~nm}$. Dessa forma, diferentes comprimentos de onda produzem efeitos diversos nos tecidos, podendo ocasionar alterações na microcirculação, regeneração tecidual e inibição de fatores inflamatórios $[6,16,17]$.

Os efeitos das irradiações com LBI são percebidos de forma local, no entanto, benefícios sistêmicos estão descritos. Devido à capacidade de bioestimulação do laser no local irradiado, os produtos gerados a partir da estimulação local passam a percorrer todo o corpo pela corrente sanguínea e fluxo linfático, garantindo que todos os sistemas sejam beneficiados com os produtos estimulados pela terapia com LBI [7,18-20].

\section{CONCLUSÕES}

A terapia com LBI ofereceu resultados positivos aos pacientes com NPH, uma vez que todos os estudos analisados destacaram diminuição da dor nos pacientes investigados. Entretanto, embora os estudos mostrem que a terapia com LBI é eficaz na redução da dor na NPH, não há um consenso quanto aos parâmetros a serem utilizados. Em razão dos diferentes protocolos empregados, é importante realizar mais estudos que auxiliem a padronizar quais os parâmetros da terapia com LBI devem ser aplicados na NPH.

\section{NOTA}

Declaração de conflitos de interesse

Os autores declaram não haver conflitos de interesse relevantes ao conteúdo deste estudo.

\section{REFERÊNCIAS}

1. Portella AVT, Souza LCB, Gomes JMA. Herpes-zoster and post-herpetic neuralgia. Rev Dor. 2013;14(3):210-15. http://dx.doi.org/10.1590/S1806-00132013000300012

2. Schmader KE, Dworkin RH. Natural history and treatment of herpes zoster. J Pain. 2008 Jan;9(1 Suppl 1):S3-9. http://dx.doi. org/10.1016/j.jpain.2007.10.002

3. Coelho PAB, Coelho PB, Carvalho NC, Duncan MS. Diagnóstico e manejo do herpes-zóster pelo médico de família e comunidade. Rev Bras Med Fam Comunidadee. 2014;9(32):279-85. http://dx.doi.org/10.5712/rbmfc9(32)994

4. Lobo IM, Santos ACL, Santos Júnior JÁ, Passos RO, Pereira CU. Vírus varicela zoster. Rev Bras Med. 2015;72(6):231-38.

5. Ferreira DC, Martins FO, Romanos MTV. Impact of low-intensity laser on the suppression of infections caused by Herpes simplex viruses 1 and 2: in vitro study. Rev Soc Bras Med Trop. 2009 Jan-Feb;42(1):82-5. http://dx.doi.org/10.1590/S003786822009000100018

6. Pedreira AF, Sá M, Medrado ARAP. O uso da terapia laser de baixa intensidade após exodontia de terceiros molares: revisão de literatura. Rev Bahiana Odonto. 2013;4(1):37-45. http://dx.doi.org/10.17267/2238-2720revbahianaodonto.v4i1.119

7. Prockt AP, Takahashi A, Pagnoncelli RM. Uso de Terapia com Laser de Baixa Intensidade na cirurgia bucomaxilofacial. Rev Port Estomatol Med Dent Cir Maxilofac. 2008;49(4):247-55. http://dx.doi.org/10.1016/s1646-2890(08)70054-2 
8. Vasconcelos Filho PO. Tratamento alternativo para neuralgia pós-herpética após transplante pulmonar. Relato de caso. Rev Dor. 2012;13(1):85-8. http://dx.doi.org/10.1590/S1806-00132012000100016

9. Abreu EMC, Nicolau RA. Terapia a laser de baixa intensidade na regeneração do tecido nervoso após lesão medular. Rev Neurocienc. 2015;23(2):297-304. http://dx.doi.org/10.4181/RNC.2015.23.02.1005.8p

10. Chung H, Dai T, Sharma SK, Huang YY, Carroll JD, Hamblin MR. The nuts and bolts of low-level laser (light) therapy. Ann Biomed Eng. 2012 Feb;40(2):516-33. http://dx.doi.org/10.1007/s10439-011-0454-7

11. Knapp DJ. Postherpetic neuralgia: case study of class 4 laser therapy intervention. Clin J Pain. 2013 Oct;29(10):e6-9. http://dx.doi.org/10.1097/AJP.0b013e31828b8ef8

12. Kneebone WJ. Therapeutic laser in the treatment of Herpes zoster. Pract Pain Manag. 2012;12(8):1-7.

13. Stump JL, Allen M, Oglesb O. Postherpetic Neuralgia Pain and Laser Acupuncture. Pract Pain Manag. 2011;11(8):1-10.

14. Valeriani M, Pazzaglia C, Cruccu G, Truini A. Clinical usefulness of laser evoked potentials. Neurophysiol Clin. 2012 Oct;42(5):345-53. http://dx.doi.org/10.1016/j.neucli.2012.05.002

15. Franz M, Spohn D, Ritter A, Rolke R, Miltner WH, Weiss T. Laser heat stimulation of tiny skin areas adds valuable information to quantitative sensory testing in postherpetic neuralgia. Pain. 2012 Aug;153(8):1687-94. http://dx.doi.org/10.1016/j. pain.2012.04.029

16. Schäfers M, Tölle TR. Aktuelle Therapie neuropathischer Schmerzen. Nervenarzt. 2013 Dec;84(12):1445-50. http://dx.doi. org/10.1007/s00115-012-3623-5

17. Cruccu G, Truini A. Neuropathic pain and its assessment. Surg Oncol. 2010 Sept;19(3):149-54. http://dx.doi.org/10.1016/j. suronc.2009.11.012

18. Fleckenstein J, Kramer S, Hoffrogge P, Thoma S, Lang PM, Lehmeyer L, Schober GM, Pfab F, Ring J, Weisenseel P, Schotten KJ, Mansmann U, Irnich D. Acupuncture in acute herpes zoster pain therapy (ACUZoster) - design and protocol of a randomised controlled trial. BMC Complement Altern Med. 2009 Aug 12;9:31. http://dx.doi.org/10.1186/1472-6882-9-31

19. Franz M, Ritter A, Puta C, Nötzel D, Miltner WH, Weiss T. Laser heat hyperalgesia is not a feature of non-specific chronic low back pain. Eur J Pain. 2014 Nov;18(10):1501-8. http://dx.doi.org/10.1002/ejp.535

20. Bispo LB. A nova tecnologia do laser terapêutico no controle da dor. Rev Bras Odontol. 2009;66:107-11. 